\title{
Percepción de la problemática de la cuenca del río Jequetepeque
}

\author{
Claudia Vasquez Miñope ${ }^{1}$ \\ Recibido: 07 julio 2021 | Aceptado: 13 noviembre 2021
}

\section{RESUMEN}

El estudio tuvo como objetivo identificar los principales problemas que la cuenca del río Jequetepeque presenta, a través del análisis de los factores bióticos, abióticos y socioeconómicos; con el fin de contribuir en el manejo integral y sostenible de la cuenca. La metodología utilizada, consistió en realizar la recopilación de información primaria y secundaria, para su posterior diagnóstico. La cuenca del río Jequetepeque se ve influenciada por su geomorfología no homogénea, el fenómeno de El Niño y la corriente peruana de Humboldt; por lo cual, las características climáticas y la vegetación muestran variación a lo largo de la cuenca. Entre la problemática identificada está el frecuente movimiento de flujos hiperconcentrados (huaycos), los cuales se intensifican en periodos de fenómeno de El Niño, afectando centros poblados, carreteras y actividades agrícolas; por ello es necesario la implementación de prácticas mecánico estructurales, prácticas agronómicas silviculturales, entre otras. Del mismo modo, el crecimiento demográfico y el uso intensivo de los suelos en cultivos y sobrepastoreo favorece el proceso de erosión; por lo cual, es importante implementar paquetes tecnológicos para cada región.

Palabras clave: agua, conflicto, diagnostico.

\footnotetext{
${ }^{1}$ Facultad de Ciencias Forestales, Universidad Nacional Agraria La Molina. Lima, Perú. 20140186@lamolina.edu.pe
} 


\section{ABSTRACT}

The study aimed to identify the main problems of the Jequetepeque River Basin, through the analysis of biotic, abiotic and socioeconomic factors in order to contribute to the integrated and sustainable management of the basin. The methodology used consisted of compiling primary and secondary information for its subsequent diagnosis. The Jequetepeque River Basin is influenced by its nonhomogeneous geomorphology, El Niño Phenomenon and the Peruvian Humboldt Current. Therefore, climatic characteristics and vegetation show variations throughout the basin. The main problems identified are the frequent movement of hyper-concentrated flows (landslide), which intensify in periods of the El Niño phenomenon, affecting populated centers, roads and agricultural activities. For that reason, it is necessary to implement structural mechanical practices, silvicultural agronomic practices, among others. Likewise, population growth and the intensive use of soils for crops and overgrazing favor the erosion process; therefore, it is important to implement technological packages for each region.

Key words: water, conflict, diagnosis.

\section{INTRODUCCIÓN}

La cuenca hidrográfica, mediante sus componentes bióticos, abióticos y socioeconómicos, nos permite relacionar y comprender la complejidad de este sistema, con el fin de proponer soluciones que permitan asegurar la provisión sostenible del agua (Rodriguez, 2018).

La cuenca del río Jequetepeque, presenta una marcada diferencia en su parte baja y alta, tanto en aspectos climáticos, actividades económicas y crecimiento demográfico. Entre las actividades antrópicas, la explotación agrícola es la que demanda mayor porcentaje de agua (Guerrero y Florián, 2013).

Entre los efectos ambientales negativos, se encuentra la ocurrencia de huaycos, deslizamientos, inundaciones, entre otros. Los cuales afectan las actividades socioeconómicas, perjudicando la calidad del agua y por ende la calidad de vida de la población de los departamentos de Cajamarca y La Libertad (SEDALIB S.A., 2018).

El presente documento descriptivo, ha sido desarrollado bajo una exhaustiva revisión de fuentes primaria y secundaria, con la finalidad de identificar la problemática de la cuenca del río Jequetepeque, a través de los componentes biofísicos y socioeconómicos de la cuenca.

\section{Ubicación y Extensión}

La cuenca del río Jequetepeque se encuentra ubicada en la parte norte del Perú, orientado en dirección este-oeste, perteneciente a la vertiente del Pacífico. Su inicio se encuentra en la Cordillera Occidental de los Andes, comprendida entre dos regiones, Cajamarca (provincias de Cajamarca, Contumazá, San Pablo 
y San Miguel) y La Libertad (provincias de Pacasmayo y Chepén). En su parte media, se encuentra el embalse Gallito Ciego, fuente de agua para el desarrollo agrario de la costa, con una capacidad de 400MMC (Millones de Metros Cúbicos) (Universidad Nacional de Ingeniería [UNI], 2000).

La cuenca está formada por un total de nueve subcuencas las cuales abarcan una superficie de 3 956,4 $\mathrm{km} 2$, una pendiente promedio de $37,6 \%$ y un perímetro aproximado de $577,2 \mathrm{~km}$. De la extensión total de la cuenca, aproximadamente el 42,29\% (1 $673,16 \mathrm{~km} 2$ ), corresponde a la cuenca húmeda. Limita por el Norte con las cuencas de los ríos Zaña y ChancayLambayeque; por el Sur con la cuenca del río Chicama y la quebrada Cupisnique; por el Este con las cuencas de los ríos Cajamarca y Llaucano y por el Oeste con el Océano Pacífico (Alcántara, 2015).

\section{COMPONENTES ABIÓTICOS}

\section{A. Geología}

En el estudio hidrogeológico, realizado por el Instituto Geológico, Minero y Metalúrgico-INGEMMET

(2006),

sostiene que las aguas subterráneas se encuentran ligadas a las condiciones geológicas de la cuenca, entre ellas la naturaleza de la roca y sus características litológicas, denominadas acuíferos y roca almacén.

Asimismo, se diferencian los tipos de acuíferos; el acuífero poroso no consolidado del piso de valle, el cual corresponde a depósitos aluviales y fluviales, de gran productividad, recarga constante y reservas potenciales, debido a la infiltración natural y artificial producto de los riegos por inundación de la actividad agrícola, por otro lado, se encuentran los acuíferos fisurados discontinuos de ladera y altura siendo el acuífero fisurado Chimú y Farrat, en este último se observan fisuras abiertas, fracturas y fallas, producto de la actividad tectónica (Peña et al., 2015).

Cabe señalar que la cuenca del Jequetepeque, se encuentra ubicada en el flanco occidental de la Cordillera de los Andes. Por lo cual, su formación es resultado de la confluencia entre la Placa Sudamericana y la Placa de Nazca. Toda la estratigrafía, estructuras, magmatismo, mineralización y sismicidad de la Cordillera de los Andes y del territorio peruano son resultado de la subducción de la Placa de Nazca por debajo de la denominada subducción andina (Mejía, 2009).

\section{B. Geomorfología}

Según Peña et al. (2015), la cuenca presenta cinco unidades morfológicas claramente diferenciadas: planicie costanera, lomadas y colinas, montañas de moderada pendiente, montañas de alta pendiente y un pequeño sector de la parte alta denominada altiplanicies.

En el estudio realizado por Alcántara (2019), en la cabecera de la cuenca del río Jequetepeque, concluyó que en la parte norte existen partes llanas con baja pendiente y que el $67 \%$ de los territorios se encuentran formados por lomas y colinas, con pendientes leves, y pequeñas planicies. En la parte media de la cabecera de cuenca, la geomorfología se va modificando levemente constituido por colinas $y$ valles, con pendientes moderadas. A 
nivel de cabecera sur, la pendiente se torna más agreste donde el $53,8 \%$ se encuentra entre fuertemente inclinado a escarpado, relacionado con el grado de concentración de humedad y vegetación. Con ello, dicho autor sostiene que la geomorfología no es homogénea a lo largo del cinturón de las cabeceras de cuenca del río Jequetepeque.

La Altitud se extiende desde el nivel del mar hasta los $4201 \mathrm{msnm}$ con una altitud promedio de $2624 \mathrm{msnm}$ (SEDALIB S.A, 2018)

\section{Clima}

Las características climáticas de la cuenca, se ve influenciada por la corriente marina de Humboldt, de aguas frías procedentes del Pacífico Sur, y por las corrientes cálidas procedentes de la zona ecuatorial, ligadas al fenómeno El Niño y por la altitud de la cordillera de los Andes (López y Giron, 2007).

El clima varía desde el oceánico subtropical de alta montaña (CWb) a climas calientes del desierto (BWh) (Climate data for cities worldwide, s.f.). Debido a ello, la precipitación varía conforme la altitud. En la zona costera, la precipitación promedio anual es de $15 \mathrm{~mm}$, existiendo incluso meses con precipitación de $0 \mathrm{~mm}$, debido a la corriente peruana de Humboldt. En años correspondientes al fenómeno de $\mathrm{El}$ Niño se ha registrado precipitaciones de 220 a 3000 mm (Tavares y Rondón, 2015). En tierras altas la precipitación promedio es de $1000 \mathrm{~mm} /$ año.

Además, la temperatura media anual en la costa, se presenta alrededor de $23^{\circ} \mathrm{C}$ con una fluctuación mensual de aproximadamente $7^{\circ} \mathrm{C}$ en el transcurso del año. En las partes superiores, las temperaturas anuales promedio se encuentran entre $8^{\circ} \mathrm{C}$ y $14^{\circ} \mathrm{C}$, con muy ligeras fluctuaciones mensuales (SEDALIB S.A, 2018).

\section{Hidrografía}

La cuenca del río Jequetepeque está conformada por tres ríos principales, 30 ríos secundarios y una red de pequeños ríos, riachuelos y quebradas en las microcuencas, generando caudales entre $230,23 \mathrm{~m}^{3} / \mathrm{seg}$ (época de lluvia) y $0,168 \mathrm{~m}^{3} / \mathrm{seg}$ (época de estío) (Peña et al., 2015). Todos estos cursos de agua se originan en las cumbres de la cordillera occidental (SEDALIB S.A, 2018).

El río Jequetepeque, río principal con un recorrido de $174,6 \mathrm{~km}$ de longitud (Naupari, 2020), resulta de la confluencia de los ríos Chilete y San Miguel, los cuales se unen a la altura del pueblo de Llallán. Aguas abajo, el río Jequetepeque recibe los aportes del río Pallac por el margen derecho y de la quebrada Chausis por el margen izquierdo (SEDALIB S.A, 2018).

En la década de los 80 , se construyó la represa Gallito Ciego, cubriendo un área de aproximadamente $14 \mathrm{~km}^{2}$, con volumen útil de 400 millones de metros cúbicos de aguas provenientes del río Jequetepeque (Metzger, 2017). La utilización de toda esta importante infraestructura hidráulica, permite el desarrollo de una intensa actividad agrícola y ganadera en los valles (Peña et al., 2015).

\section{E. Suelos}

Según López y Girón (2007), de acuerdo a su origen, los suelos de la 
aluviales, residuales:

- Los fluviales, son originados por la deposición de materiales arrastrados por el río Jequetepeque, Pallac y San Miguel (Puclush), los cuales forman terrazas al encontrar zonas de pendientes suaves.

- Los aluviales, son productos de la deposición de materiales arrastrados por las corrientes de agua de poco recorrido que se realizaron en ambientes de relieve suave, formando depósitos aluviales.

- Los coluvio-aluviales, son originados por la deposición de materiales arrastrados por las aguas en zonas de pendiente pronunciada, con escaso recorrido, formando depósitos conocidos como piedemontes y conos de deyección.

- Los residuales, son productos de la meteorización (descomposición) de las rocas, las cuales, por acción de la variación de la temperatura, en la zona árida y por reacción de los elementos minerales, en la parte húmeda, se van desmenuzando cada vez más hasta formar el suelo.

El mismo autor señala que de acuerdo al tipo de suelo, se encuentra: Andosol, Kaztanozem, Leptosol, Gleisol, Regosol, Phaeozem, Miscelaneo Cauce y Miscelaneo Mina.

\section{F. Capacidad de Uso Mayor de los Suelos}

En el diagnóstico hídrico rápido de la

cuenca del rio Jequetepeque como fuente de agua y servicios ecosistémicos hídricos para la EPS SEDALIB S.A. (2018) se registra la siguiente información:

En la parte baja, la aptitud de las tierras para el riego está condicionada por las limitaciones presentes de los suelos, topografía y drenaje, que se reflejan en los costos de desarrollo y en la productividad de las mismas tierras. La capacidad de uso mayor de los suelos en esta parte, está referida a una superficie de 29788,85 ha, dividido en dos categorías que representan a las diferentes zonas edáficas y la vocación potencial de las tierras.

En la parte alta, el suelo está referido a una superficie aproximada de 407 100,53 ha, en las que se han determinado cuatro clases de Capacidad de Uso Mayor de los Suelos: cultivo en limpio $(A)$, pastos $(P)$, producción forestal $(F)$ y protección $(X)$.

\section{Uso Mayor del Suelo}

Parte Baja

Tierras aptas para cultivos en limpio

Transitorios

Permanentes

Tierras aptas para pastos

Parte Alta

Tierras aptas para cultivos en limpio

Tierras aptas para pastos

$44726,23 \quad 10,98$

Tierras de protección

359494,30

99,60

28705,9

964

119,00

0,40

407100,53

$2880,00 \quad 0,71$
Tabla 1.

Uso mayor de suelos en la parte baja y alta de la Cuenca Jequetepeque. Fuente: SEDALIB

S.A., 2018 


\section{COMPONENTES BIÓTICOS}

\section{A. Flora}

En la parte alta de la cuenca, zona denominada jalca, existen áreas aisladas con bosques naturales $y$ bosques desarrollados con plantaciones de eucalipto y pino; gran parte de esta zona está cubierta con pastizales nativos, como el "ichu" (Stipa ichu (Ruiz \& Pav.) Kunth) de uso común en el pastoreo (Alcántara, 2019).

Así mismo, en la cabecera norte, donde existe menor pendiente, se observa mayor concentración de humedad y vegetación permanente de herbáceas, arbustivas y arbóreas como "aliso" (Alnus acuminata Kunth) y "queñual" (Polylepis racemosa Ruiz \& Pav.). En la parte sur, la vegetación es más dispersa y escasa (Alcántara, 2019).

A 3000 y 3850 msnm se encuentra la Granja Porcón, la forestación y reforestación con pinos ha generado un mejoramiento de microclima en 10 966 ha, lo cual ha mejorado la producción de semillas de papa, cultivos de hongos y alcachofas, etc., además se realiza el aprovechamiento forestal de dicha especie para la artesanía e industrialización (Peña et al., 2015).

La vegetación herbácea, es la más abundante, y sirve como acolchonamiento de la velocidad con que impacta las gotas de agua en el suelo, al mismo tiempo que la retiene y la dirige en dirección del tallo y raíces, lo cual ayuda a aumentar la capacidad de infiltración hasta su saturación, si es que la intensidad de lluvia continua; caso contrario, las aguas infiltradas van percolando y originando acuíferos profundos 0 aguas subsuperficiales (Alcántara, 2015).

Con respecto a la flora de los bofedales, se reporta la presencia de Alchemilla diplophylla Diels, Alchemilla pinnata Ruiz \& Pav; además de Cyperus L. "totora”, Scirpus rigidus Boeckeler, Plantago major L. "llantén", Calamagrostis curvula (Wedd.) Pilg., Rumex crispus L. "mala yerba", esta última tiene la propiedad de almacenar agua y es usada para la recuperación de bofedales (MINAGRI, 2015).

En las orillas de los ríos, se encuentran especies arbóreas nativas como: Escallonia pendula (Ruiz \& Pav.) Pers. "pauco", $P$. racemosa "queñual”, $A$. acuminata "aliso", Escallonia paniculata (Ruiz \& Pav.) Roem. \& Schult. "chachacoma", Salix humboldtiana Willd. "sauce", Cedrela montana Moritz ex Turcz. "cedro". Entre las especies exóticas de mayor importancia que crecen en la orilla del río se tiene al Eucalyptus globulus Labill. “eucalipto" y Pinus L. “pino".

\section{B. Zonas de Vida}

Según el diagnóstico hídrico realizado para la Empresa Prestadora de Servicio SEDALIB S.A. (2018), la cuenca del río Jequetepeque presenta 15 zonas de vida.

\begin{tabular}{cccc}
\hline Simbología & Descripción & Área (ha) & $\%$ \\
\hline bh-MBT & $\begin{array}{c}\text { Bosque húmedo- } \\
\text { Montano Bajo } \\
\text { Tropical }\end{array}$ & 40178,9 & 10,16 \\
& &
\end{tabular}




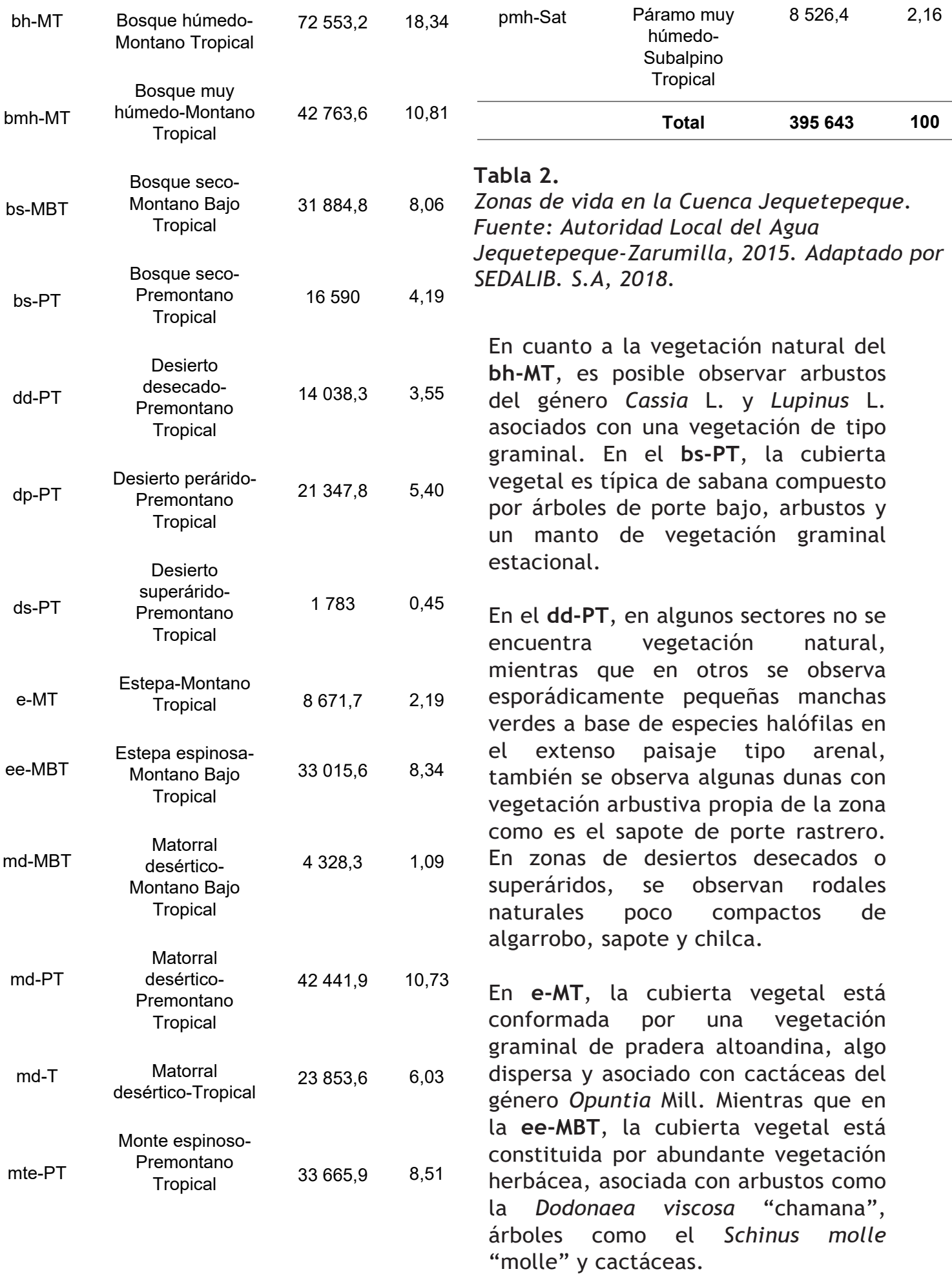


En el md-PT y en el md-T, la cubierta vegetal está conformada por especies arbóreas como el sapote, el algarrobo y arbustos como el Capparis spicata. También, las cactáceas están presentes, siendo el género Neoraimondia Britton \& Rose. de porte columnar prismático gigante, un indicador de esta Zona de Vida.

Finalmente, en el Páramo muy húmedo - Subalpino Tropical (pmhSaT). La cubierta vegetal lo conforma una vegetación típica de pradera alto andina, constituida por pastos naturales provenientes de diversas familias, principalmente de la familia Poaceae (gramíneas); en general esta zona tiene una composición florística compleja y es más densamente poblada.

\section{Fauna}

En el estudio de Evaluación De Recursos Hídricos En La Cuenca del Rio Jequetepeque realizado por MINAGRI (2015), identificó 3 ecosistemas:

En lagunas se encontró una diversidad de avifauna Spatula cyanoptera Vieillot, 1816 "pato colorado", Larus serranus Tschudi, 1844 "gaviota andina", Anas flavirostris Vieillot, 1816 "pato andino cabecinegra”, Anas georgica Gmelin, 1789 "pato jerga", Plegadis ridgwayi Allen, 1876 "ibis de puna"; Gallinago andina Taczanowski, 1875 "quecheche chico", entre otras aves acuáticas. La especie piscícola encontrada fue Oncorhynchus mykiss Walbaum, 1792 "trucha”.

En el caso de los bofedales; a pesar de que se encuentren alterados y desplazados por la actividad agropecuaria (presencia de pastos cultivados). Se observó algunas especies como Phalcoboenus megalopterus (Meyen, 1834) "chinalinda", Colaptes rupicola d 'Orbigny, $1840 \quad$ "cargacha, cortarrera", Geospizopsis plebejus (Tschudi, 1844) "fringilo pechicenizo", Colibri coruscans (Gould, 1846) "colibrí, quinde" y Bufo cophotis Boulenger, 1900 “sapo”. La presencia de aves en estos humedales, se redujo en comparación a otras cuencas, debido a la alteración de las condiciones naturales de los bofedales.

En los ríos se ha observado la presencia de aves, siendo un ambiente que sirve de bebederos o fuente de alimento de: Falco sparverius Linnaeus, 1758 “cernícalo americano", Zonotrichia capensis (Müller, 1776) "gorrión andino", Turdus chiguanco Lafresnaye \& d 'Orbigny, 1837 “chiguaco", Nycticorax Linnaeus, 1758 "huaco común". En algunos tramos del recorrido del río San Miguel, hay presencia de 0 . mykiss "trucha" (parte alta de la cuenca). Mientras que, en la zona baja; se observa la presencia de Egretta thula (Molina, 1782) "garza blanca chica", Ardea alba Linnaeus, 1758 "garza blanca grande" y Mimus longicaudatus Tschudi, 1844 "chisco".

\section{FACTORES SOCIOECONÓMICOS}

\section{A. Demografía}

Como se ha mencionado anteriormente, la cuenca de Jequetepeque se 
encuentra distribuida entre los departamentos de La Libertad y Cajamarca, abarcando un total de seis provincias y 30 distritos.

La tasa de crecimiento de la población de $1993 \quad(253 \quad 812$ habitantes) a 2017 (507 609 habs.) es de $99,9 \%$. La provincia de Cajamarca concentra la mayor población $(50,4 \%)$, mientras que las demás provincias de la parte alta presentan menor población que las zonas bajas, siendo el caso de Chepén y Pacasmayo con $20,3 \%$ y $15,4 \%$ respectivamente.

Así mismo, en comparación del censo de 2007 (179 953 habs.) y el censo del 2017 (181 315 habs.), la zona costera presenta una tasa de crecimiento de 0,76\% (Instituto Nacional de Estadística e Informática [INEI], 2018).

\section{B. Distribución urbana-rural}

En la cuenca del río Jequetepeque, $66,1 \%$ de los habitantes viven en el sector urbano, mientras que el 33,9\% de la población es rural. Esta situación se debe a la concentración poblacional en las ciudades costeras como Chepén (88\%) y Pacasmayo $(94,5 \%)$. En las zonas altas, la situación es inversa para las provincias de San pablo, San Miguel y Contumazá, donde principalmente la población es rural. Sin embargo, la provincia de Cajamarca es la excepción ya que el $61,6 \%$ de su población total es urbana (INEI, 2018).

\begin{tabular}{ccccc}
\hline $\begin{array}{c}\text { Departamento } \\
\text { Provincia }\end{array}$ & $\begin{array}{c}\text { Población } \\
\text { Rural }\end{array}$ & $\begin{array}{c}\text { Población } \\
\text { Urbana }\end{array}$ & Total & $\%$ \\
\hline Cajamarca & $\mathbf{1 5 7 0 4 3}$ & $\mathbf{1 6 9 2 5 1}$ & $\mathbf{3 2 6 2 9 4}$ & $\mathbf{6 4 , 3}$ \\
Cajamarca & 98207 & 157413 & 255620 & 50,4 \\
San Pablo & 12563 & 2548 & 15111 & 3,0 \\
San Miguel & 31486 & 3379 & 34865 & 6,9 \\
Contumazá & 14787 & 5911 & 20698 & 4,1 \\
La Libertad & $\mathbf{1 5 1 0 1}$ & $\mathbf{1 6 6 2 1 4}$ & $\mathbf{1 8 1 3 1 5}$ & $\mathbf{3 5 , 7}$ \\
Pacasmayo & 5657 & 97240 & 102897 & 20,3 \\
Chepén & 9444 & 68974 & 78418 & 15,4 \\
TOTAL & $\mathbf{1 7 2 1 4 4}$ & $\mathbf{3 3 5 4 6 5}$ & $\mathbf{5 0 7 6 0 9}$ & $\mathbf{1 0 0}$ \\
\hline
\end{tabular}

Tabla 3.

Distribución población urbana-rural de la Cuenca del río Jequetepeque. Fuente: INEI, 2017.

Según información del Consorcio para el Desarrollo de la Ecorregión Andina (CONDESAN) la población del valle del Jequetepeque es pobre. Un $75 \%$ de la población son campesinos de tierra seca y cuentan con 2,5 ha (SERVINDI, 2015).

\section{Distribución y Tenencia de la tierra}

En la zona alta existe presencia de un sector campesino cuyas posesiones de tierra son sumamente pequeñas. El 73\% de familias cuentan con menos de 5 hectáreas de terreno, incluyendo un $29,3 \%$ con áreas no mayores a 1,0 hectárea. Un 9,1\% adicional de familias campesinas carecen de tierras y sólo un $1,9 \%$ tiene propiedades mayores a 5 hectáreas (UNI, 2000). 
En la zona baja el $69 \%$ de las familias disponen del $37 \%$ del área agrícola del Valle, presencia que ha sido incrementada considerablemente después del proceso de parcelación. Los propietarios medianos (10 a 20 hectáreas) y grandes (más de 20 hectáreas) utilizan en promedio el $16,2 \%$ de las tierras agrícolas del valle (UNI, 2000).

\section{Migración}

Respecto a los movimientos migratorios de la población, Villanueva (2017), nos menciona que la cuenca alta del Jequetepeque se caracteriza principalmente por el predominio de los procesos de emigración hacia ciudades costeras debido a la ausencia de oportunidades educativas y de empleo. Entre ellas se encuentra a las ciudades de Trujillo, Pacasmayo, explicado por su condición de puerto y por ser la sede del mayor complejo industrial existente en el Valle (la empresa Cementos Norte S.A.) y Chepén, debido a su dinamismo comercial gracias a su ubicación estratégica.

\section{E. Actividades económicas}

El uso de la tierra varía significativamente según las zonas. En el valle o zona baja la superficie agrícola alcanza un 41,1\%, mientras que en la zona alta es de sólo 16,8\%. En la zona alta predominan los pastos naturales, siendo un 36,1\% del total frente al $1,1 \%$ de la zona baja. Las tierras marginales y forestales representan el $47,1 \%$ en la zona alta $y$ el $57,8 \%$ en la zona baja (SEDALIB S.A., 2018).
La actividad agrícola en tierras bajo riego se reduce en las riberas delJequetepeque, o a pequeñas zonas en las laderas. Sólo un 40,37\% del área agrícola total dispone de riego en toda la cuenca, frente al 59,62\% en condiciones de secano (SEDALIB S.A., 2018).

\section{Zona Alta}

El uso es para el pastoreo de ganado vacuno y ovino a pequeña o mediana escala y el cultivo de papas y otros tubérculos andinos, granos, maíz y leguminosas. En esta zona escasea el agua, teniendo al área agrícola bajo riego con menos del $12 \%$ del total, frente al $88,27 \%$ de secano. A ello se debe agregar el hecho de que alrededor de las 3/4 partes de las tierras de secano en esta zona se hallan en descanso, lo que limita notablemente su capacidad productiva (UNI, 2000).

El trigo es el cultivo de mayor importancia. La mayor parte de las cosechas se destina al consumo familiar, usado para la elaboración de harinas. Sobresalen en su producción los distritos de Chetilla, San Miguel, Llapa, El Prado y Contumazá. El Maíz amiláceo, es el segundo cultivo en importancia, predominando en los sistemas de producción campesina, destinándose mayormente al autoconsumo. Los principales distritos productores de maíz son Calquis, Cochán y Llapa, así como Contumazá, Asunción y Chetilla (UNI, 2000).

La utilización del suelo para la producción de productos agrícolas, tales como la papa, la oca y el olluco, ha ido modificando el relieve en su 
tendencia de convertirlo en un espacio plano, asimismo, en el proceso del movimiento de tierras se ha generado un flujo de fluidos, aumentando la porosidad, por lo que estos espacios son un elemento importante de infiltración y alimentación de agua subterránea (Alcántara, 2019).

Otra actividad realizada es la minería, encontrándose en la provincia de Cajamarca, la empresa Yanacocha, el cual posee el yacimiento más importante de oro en la sub-región (UNI, 2000).

\section{Zona Baja}

El uso de la tierra es para el cultivo de caña de azúcar, arroz, frutas y verduras. Siendo, el arroz el principal producto por su rentabilidad y extensión cultivada desde hace varias décadas. Se trata de un cultivo intensivo que usa tecnología moderna y se destina al mercado nacional. Los principales centros arroceros del valle son: Guadalupe, San Pedro, Limoncarro, Pacanga y Chepén (Villanueva, 2017).

La caña de azúcar, es un producto usado para elaborar chancaca, aguardiente y miel de caña, bienes con demanda local. En los últimos años, la extensión del cultivo de caña se ha incrementado en algunos distritos. Además, en la provincia de Contumazá se explota artesanalmente la arcilla de Cascas y en Yonán el carbón de piedra, rocas calizas y arcillosas, siendo importante la presencia de las calizas que explota Cementos Pacasmayo en Tembladera (UNI, 2000).

\section{F. Uso del agua}

El uso total del agua en la cuenca llega a $727 \quad 186 \quad 000 \quad \mathrm{~m}^{3}$ y está representada por los sectores agrícola, poblacional, pecuario e industrial; de los cuales, el uso agrícola es el que obtiene una mayor demanda, siendo mayor a 719,410 MMC anuales constituyendo el $98 \%$ del total de agua de la cuenca (Guerrero y Florián, 2013).

En el uso pecuario, el vacuno es el principal consumidor con $647000 \mathrm{~m}^{3}$ por año, le sigue el ganado equino con $256000 \mathrm{~m}^{3}$ y el ganado ovino con $107000 \mathrm{~m}^{3}$. El uso total de agua por este rubro alcanza los 1,258 MMC que representa el 0,30\% del agua de la cuenca.

En la actividad industrial, los referentes a la fabricación de productos minerales no metálicos son los que tienen el mayor consumo de agua, llegando en este caso al 92,5\% del total consumido por este concepto, es decir el uso industrial llega aproximadamente a $601000 \mathrm{~m}^{3}$ por año que representa el 0,1\% del agua de la cuenca (SEDALIB S.A. ,2018).

\section{ANÁLISIS DE LA PROBLEMÁTICA}

Dentro de los factores abióticos más relevantes se encuentra su geología y geomorfología variada, ya que la cuenca presenta planicies costeras, valles, colinas, laderas y montañas, definiendo comportamientos variados tanto en clima como en suelo. Dentro de sus parámetros geomorfológicos, la cuenca del río Jequetepeque presenta un factor de forma de 0,13 (Naupari, 2020), es decir se trata de una cuenca 
de forma alargada, en donde las descargas o crecientes son de menor volumen (Verdugo, 2019); sin embargo, la presencia de periodos del Fenómeno El Niño ocasiona movimientos en masa, deslizamientos, derrumbes, etc. ocasionando problemas de erosión en las laderas, acumulación de sedimentos, inundaciones en centros poblados y áreas agrícolas (Peña et al, 2015).

Asimismo, desde el punto de vista geológico los acuíferos presentes en la cuenca vienen siendo usados mediante afloramientos en forma natural (manantiales, aniegos, bofedales, etc), así como artificiales (pozos, sondeos, galerías filtrantes, pantallas de regulación) (Peña et al, 2015). Sin embargo, su uso ha sido limitado en el valle y por ende se cuenta con escaza información del movimiento y circulación de las aguas subterráneas de la parte alta.

En cuanto a los factores bióticos, a pesar de que la presencia de la granja Porcón ha contribuido como recarga artificial de acuíferos, existe deforestación en grandes sectores de la cuenca debido a actividades agrícolas que vienen alterando la vegetación natural y fauna. Siendo los bofedales, los ecosistemas más afectados.

Según la política de Estado $\mathrm{N}^{\circ} 34$ sobre ordenamiento y gestión territorial alcanzado en el Foro del Acuerdo Nacional en el año 2002 (sociedad civil, Estado y partidos políticos), se busca implementar mecanismos de gestión, para la inversión de proyectos y servicios a la ciudadanía, entre ello obras civiles para captación, almacenamiento y distribución del agua, para expansión urbana o rural y de recuperación de suelos (Centro Nacional de Planeamiento Estratégico [CEPLAN], 2002). Por ello, es importante que la cuenca de Jequetepeque maneje una zonificación de acuerdo a sus componentes bióticos y abióticos, con la finalidad de implementar prácticas mecánico estructurales. Así mismo, AGRORURAL (2011) menciona como alternativas para la conservación de suelos de laderas la rehabilitación de andenes, implementación de terrazas de formación lenta, zanjas de desviación o infiltración, estabilización de quebradas, ente otros. En la cuenca se tiene como ejemplo al distrito de Encañada (Cajamarca) donde se han realizado 600 ha de terrazas de formación lenta asociado con especies leñosas, con la finalidad de controlar la humedad y el grosor de los suelos para la funcionalidad de cultivos agrícolas (Tapia y Sanchez, 1996 citado por Iberico, 2015). Además, según el Modelo Negromayo localizado en la parte Sur Oeste de la ciudad de Cajamarca, se ha identificado obras civiles como acequias de infiltración, también conocidas como zanjas de infiltración con plantaciones de $E$. globulus, Pinus patula y $P$. radiata, con la finalidad de captar agua de la escorrentía superficial y permitir su infiltración para controlar la erosión de suelos.

De igual forma, se debería tomar en cuenta los buenos resultados obtenidos a través de paquetes tecnológicos como sistemas agroforestales, principalmente silvopastoriles, los cuales han promovido una disminución de la 
físicas, químicas y biológicas del suelo, aumentando la cobertura vegetal, diversidad florística, la producción agrícola y el ingreso económico de la población (AGRORURAL, 2011).

A pesar del impacto negativo que tiene el fenómeno El Niño, Peña et al. (2015) menciona que se debe buscar oportunidades para retener las aguas y almacenarlas en el subsuelo dentro de los reservorios acuíferos, ya que la cuenca presenta importantes acuíferos donde se puede aprovechar el agua subterránea mediante galerías filtrantes.

A nivel social, el crecimiento poblacional sumado a ello las condiciones de vida dentro de los centros poblados ha ocasionado movimientos migratorios hacia ciudades costeras ocasionando una mayor demanda de agua, siendo su principal abasto las aguas superficiales, por ello es importante el suministro de aguas subterráneas sobre todo en las partes altas donde se práctica una agricultura de secano. Por otro lado, en la cuenca alta existen diversas reservas minerales, dentro de ellas se encuentra la empresa minera Yanacocha. En la cual se ha presentado una sería de luchas constantes entre los mineros y campesinos, donde estos últimos buscan defender sus ríos de la contaminación por parte de la minera (Saade, 2013). Teniendo finalmente a ambas partes en disputa por el acceso al agua en cantidad y calidad. Por ello, debemos instar en la política de Estado $\mathrm{N}^{\circ} 33$, la cual hace hincapié sobre los recursos hídricos para la protección y aprovechamiento de las aguas en las ciudades y zonas rurales del país (CEPLAN, 2002), con la finalidad de tener un mayor control sobre la extracción de dichos materiales.

A nivel ambiental, la actividad minera mediante los relaves aumenta la concentración de contaminantes en el agua (metales pesados), perjudicando a la población que capta el agua de los ríos cercanos o que realiza la actividad de pesca. Sumado a ello existen rellenos sanitarios a lo largo del territorio que no cumplen con las características necesarias para evitar la contaminación del agua subterránea, se han reportado además vertimientos de aguas residuales domésticas provenientes de los diversos poblados asentados cerca al cauce del rio Jequetepeque (Peña et al., 2015). En la actividad agrícola, el uso desmedido de plaguicidas e insecticidas en cultivos ha afectado el hábitat de peces y la flora acuática. A esto se suma el quemado de residuos agrícolas, los cuales al mezclarse con las aguas de riego salinizan el agua que se infiltra en el subsuelo y que llegan al acuífero. Por lo mencionado, es importante tener un adecuado control en el piso de valle, donde el acuífero es poroso y permeable, por ende, tiene mayores posibilidades de que los elementos contaminantes lleguen a ellos (Peña et al., 2015).

A nivel socio-económico, la gran cantidad de pequeñas parcelas con sobrepastoreo en la parte alta de la cuenca, perjudican la captación de agua y por ende el suministro para las zonas medias y bajas a lo largo del año. Del mismo modo, favorece el proceso de erosión hídrica de los suelos 
y el transporte de sedimentos. Avizorando que la cuenca carece de un manejo racional con respecto a sus pastos naturales. No existen planes que consideren la capacidad de carga ni prácticas para su conversación o recuperación. Para ello, es importante promover la agroforestería y las actividades silvopastoriles, mediante el uso de barreras vivas o sombra para el ganado. Para lograr ello, es importante promover las investigaciones sobre los pastos naturales y su distribución en la parte alta de la cuenca, con el fin de utilizar las especies aptas para el consumo del ganado, como es el caso del ecosistema de bofedales, donde se debe de incitar a emplear la especie $R$. crispus “mala yerba”.

Desde el punto de vista de las actividades económicas. En la zona costera, la escasez de precipitaciones y la gran concentración de población, ocasiona un aumento de la demanda de agua en cultivos, principalmente en las épocas de estiaje, pues esta zona depende directamente de la ocurrencia de lluvias en la parte alta. Se debe además precisar que esta zona realiza un uso intensivo de los suelos en cultivos como arroz y caña de azúcar. Así mismo, se registran problemas de salinización de los suelos, debido al aumento del nivel freático, producto de un inadecuado manejo del riego asociados al cultivo del arroz. Una alternativa para mejorar ello, es favorecer cultivos que demanden poca cantidad de agua y proporcionen una cobertura ante el impacto de la lluvia, como es el caso del trigo, cuya producción nacional no satisface la demanda interna, siendo necesario su importación.
Según la revista digital de la Cámara de Comercio de Lima (La Cámara, 2021) para el 2020 la producción nacional de trigo apenas representó el $8 \%$ del consumo en el país. Asimismo, un cultivo en aumento que está presente en áreas mayores a las del trigo es la cebada, insumo básico de la industria maltero-cervecera. Delgado (2021), menciona que existe una demanda creciente de cebada malteada, debido al crecimiento de las microcervecerías y al despegue de las cervezas artesanales en el Perú, especialmente en provincias con gran desarrollo de turismo. De igual manera, cada provincia debe buscar otras alternativas económicas durante el tiempo de estiaje, como es el caso de Cajamarca que actualmente posee una producción de leche competitiva. Un producto interesante para la zona costera sería la producción y comercio de la tara, pues tiene una rápida producción (cuarto año) de la cual es posible obtener taninos, gomas, hidrocoloides y galactómanos, los cuales se utilizan como aditivos o materia prima en muchas industrias.

Finalmente, a pesar de los esfuerzo realizados y de contar con un Plan de Gestión de la Oferta del Agua en el 2002 y un Plan de Ordenamiento Ambiental de la Cuenca del Río Jequetepeque para la proyección del reservorio Gallito Ciego y su valle agrícola en el 2005, donde este último presenta los lineamientos para lograr el desarrollo social, en función a las ventajas comparativas y competitivas en la cuenca media y alta del río Jequetepeque (Peña et al., 2015), Villanueva (2017) menciona que la cuenca Jequetepeque no presenta una gestión sostenible e integral del agua. Además, Guerrero-Padilla (2014), 
resalta como uno de los problemas para la gestión integral del agua el concepto de pluralismo normativo y la valoración multidimensional. El mismo autor menciona que es importante contar con un Plan de Gestión de los Recursos Hídricos (PGRHC), donde se promueva la gestión y el desarrollo coordinado del agua, la tierra y los recursos relacionados, con el fin de maximizar el bienestar social y económico resultante de manera equitativa, sin comprometer la sostenibilidad de los ecosistemas vitales (Asociación Mundial de Agua, 2008 citado por Villanueva, 2017). Para ello, se requiere un marco legal, institucional y financiero que reconozca las dimensiones de la GIRH, con la finalidad de asegurar la sostenibilidad de los recursos hídricos (GuerreroPadilla, 2014).

\section{CONCLUSIONES}

La geomorfología variante de la cuenca del río Jequetepeque, es un parámetro de gran influencia en el escurrimiento rápido o lento del agua superficial e infiltración. Lo cual, junto con el fenómeno de El Niño, incrementan los procesos de deslizamientos, inundación y erosión.

El continuo crecimiento de la población, principalmente de la zona baja y en la provincia de Cajamarca, genera una mayor demanda de agua, debido al aumento en la ocupación del territorio para cultivos de gran consumo de este recurso.

En la zona alta, la falta de estudios sobre pastos naturales y otras especies vegetales nativas, el sobrepastoreo, los conflictos sociales con empresas mineras y la predominancia de una agricultura de secano, perjudica la capacidad productiva de esta zona y su correcta recepción y almacenamiento de agua.

\section{AGRADECIMIENTOS}

A la profesora Rosa María Hermoza por las sugerencias y revisión del presente documento.

\section{LITERATURA CITADA}

Alcántara, F. A. (2015). Modelo de Geoformaciones Cóncavas para recargas de agua subterránea en cabeceras de Cuenca del Río Jequetepeque, Cajamarca [Tesis PhD, Universidad Nacional Agraria La Molina]. Repositorio Institucional UNALM. http:// repositorio.lamolina.edu.pe/ bitstream/handle/UNALM/2156/P10A42-T.pdf?sequence $=1$ \&isAllowed $=y$

Alcántara, A. (2019). Influencia de la Geomorfología y la Meteorización en la Humedad del suelo y recargas de agua en la cabecera de cuenca del río Jequetepeque. Rev. del Instituto de Investigación FIGMMG-UNMSM. 22(43), 99-106. https:// revistasinvestigacion.unmsm.edu.pe/ index.php/iigeo/article/

view/16694/14257

Delgado, M. M. (2021). Factores determinantes de la producción de cebada malteada en el Perú para productores de cerveza artesanal, Arequipa, 2019. [Tesis de maestría, Universidad Nacional Agraria La Molina]. Repositorio Institucional UNALM. 
https: / / repositorio.lamolina.edu.pe/ bitstream/handle/UNALM/4738/ delgado-montoya-maria-milagros.pdf? sequence $=1$ \&isAllowed $=y$

Centro Nacional de Planeamiento Estratégico (CEPLAN) y Foro de Acuerdo Nacional. (2002). Las 35 políticas de estado del acuerdo nacional. https: / /

www.ceplan.gob.pe/politicas-yplanes/

Climate data for cities worldwideCLIMATE-DATA. (s. f.). Consultado el 15 de junio del 2021. https:// es.climate-data.org/

Guerrero, A., y Florián, J. (2013). Demanda y uso de agua en los sectores de riego de la cuenca baja del río Jequetepeque (La Libertad, Perú). REBIOL. 33(1), 1-17. https:// revistas.unitru.edu.pe/index.php/ facccbiol/article/view/135/116

Guerrero-Padilla, A. (2014). Gestión integrada de recursos hídricos de la cuenca del río Jequetepeque, Perú. SCIENDO. 17(2).

Iberico, G. (2015). Evaluación del Modelo Negromayo en la Recuperación de la Diversidad Vegetal en las Laderas Erosionadas del Valle de Cajamarca. [Tesis de maestría, Universidad Nacional de Cajamarca]. Repositorio Institucional UNC. https: / / repositorio.unc.edu.pe/ bitstream/handle/UNC/1642/TESIS\% 20GUSTAVO\%20IBERICO.pdf? sequence $=1$ \& is Allowed $=y$
Instituto Nacional de Estadística e Informática, INEI. (2018). Resultados definitivos de los Censos Nacionales 2017- La Libertad. https:// www.inei.gob.pe/

Instituto Nacional de Estadística e Informática, INEI. (2018). Resultados definitivos de la Población Económicamente Activa 2017Cajamarca. https:// www.inei.gob.pe/

Instituto Geológico, Minero y Metalúrgico, INGEMMET. (2006). Primer Reporte de las Zonas Críticas por peligros Geológicos en la Cuenca Del Río Jequetepeque, Perú. https:// www.ingemmet.gob.pe/

López, F., y Giron, E. (2007). Informe Análisis Biofísico (Modelo Swat) Parte 1: General Cuenca Del Río Jequetepeque Cajamarca, Perú. Proyecto Pago Por Servicios Ambientales Para Generar Una Nueva Dinámica De Desarrollo Rural En Los Andes.

Mejía, J. (2009). Geología y categorización de recursos geológicos mineros del proyecto Invicta provincia de Huaura, Lima. [Tesis de pregrado, Universidad Nacional de Ingeniería]. Repositorio institucional UNI. http://cybertesis.uni.edu.pe/ handle/uni/11

Metzger, J. (2017). Modelamiento Hidrológico para Pronóstico estacional de caudales del río Jequetepeque. Servicio Nacional de Meteorología e Hidrología del Perú (SENAMHI) 
Ministerio de Agricultura y Riego y Autoridad Administrativa del AguaAAA Jequetepeque Zarumilla. (2015). Estudio Evaluación de los Recursos Hídricos en la Cuenca del río Jequetepeque. Dirección de Conservación y Planeamiento de Recursos Hídricos.

Naupari, J. R. (2020). Simulación Hidrológica del sistema regulado para evaluar las reglas de operación que permitan un manejo adecuado de la Cuenca Jequetepeque. [Tesis de pregrado, Universidad Nacional Agria La Molina]. Repositorio Institucional UNALM. https:// repositorio.lamolina.edu.pe/

bitstream/handle/UNALM/4524/

naupari-asencios-joel-rostail.pdf?

sequence $=1 \&$ isAllowed $=y$

Peña, F., Carpio, J., y Vargas, V. (2015). Hidrogeología de la cuenca de los ríos Jequetepeque (13774) y Chamán (137752). Regiones Cajamarca, La Libertad y Lambayeque. INGEMMET. Boletín, Serie H: Hidrogeología, 4, 296. https: / /

hdl.handle.net/20.500.12544/371

Programa de Desarrollo Productivo Agrario Rural, AGRORURAL. (2011). Prácticas de conservación de suelos en laderas. Lima. 247 p.

Revista Digital de la Cámara de Comercio de Lima. (2021, 11 de junio). ¿Cuánto crecieron las importaciones peruanas de trigo y maíz entre enero y abril?. La Cámara. https: / / lacamara.pe/cuantocrecieron-las-importaciones-peruanasde-trigo-y-maiz-entre-enero-y-abril/
Rodriguez, D. A. (2018). Caracterización Biofísica y Análisis de Amenazas para la cuenca del Río Monquirá como insumo en la fase de diagnóstico del plan de ordenación y manejo de Cuenca Hidrográfica (POMCA). [Tesis de grado, Universidad Pedagógica y Tecnológica de Colombia]. Repositorio https:// repositorio.uptc.edu.co/ bitstream/001/2509/1/TGT-1095.pdf

Saade, M. (2013). Desarrollo minero y conflictos socioambientales: los casos de Colombia, México y el Perú. Macroeconomía del Desarrollo 137, Naciones Unidas Comisión Económica para América Latina y el Caribe (CEPAL). https: / / repositorio.cepal.org/bitstream / handle/11362/5369/LCL3706_es.pdf

SEDALIB S.A. (2018). Diagnóstico hídrico rápido de la Cuenca del Rio Jequetepeque como fuente de agua y servicios ecosistémicos hídricos para la EPS SEDALIB S.A. http:// Www.sedalib.com.pe/upload/

drive/32019/20190305-5941539840.pdf

SERVINDI. (2015). Perú: Encuentro debatirá situación de la cuenca del Jequetepeque. https:// www.servindi.org/

Tavares, C., y Rondón, G. (2015). Impacto de la Represa de Gallito Ciego en la estabilidad de línea de costa en la desembocadura del río Jequetepeque, Perú. Espacio y Desarrollo. (27), 79-101. 
Universidad Nacional De Ingeniería e Instituto De Minería $Y$ Medio Ambiente. (2000). Estudio De Evaluación Ambiental Territorial y de Planteamientos para la reducción de la contaminación de origen minero en la Cuenca del río Jequetepeque. Perú.

Verdugo, M. (2019). Estudios morfométricos y Análisis de Inundación del rio Cumbe, provincia del Azuay; mediante los sistemas de información geográfica (ArcGIS) con la herramienta GEORAS y el programa HECRAS. [Tesis de maestría, Universidad de Alcalá]. Repositorio Institucional UAH. https:// ebuah.uah.es/xmlui/bitstream/ handle/10017/41954/

TFM_Verdugo_Cardenas_2019.pdf;jses sionid $=20048684$ E2C700ED26B2351EOB 943BDB? sequence $=1$

Villanueva, P. R. (2017). Limitaciones de la gestión del agua en la Cuenca Jequetepeque. Bases para la gestión integrada de los recursos hídricos. [Tesis de pregrado, Pontificia Universidad Católica del Perú]. Repositorio PUCP. https:// tesis.pucp.edu.pe/repositorio/ bitstream/ handle/20.500.12404/9819/ VILLANUEVA_VILLANUEVA_LIMITACION ES_DE_LA_GESTION_DEL_AGUA_EN_LA _CUENCA_JEQUETEPEQUE.pdf? sequence $=1$ \& is Allowed $=y$ 